\title{
Devastating Salt-wasting Crisis in a Four-month-old Male Child with Congenital Adrenal Hyperplasia, Highlighting the Essence of Neonatal Screening
}

Nagaspurthy Reddy ${ }^{1}$, Sucheta Sharma ${ }^{2}$, Mainak Das ${ }^{3}$, Ashutosh Kapoor ${ }^{4}$, and Upasana Maskey $^{5}$

${ }^{1}$ MediCiti Institute of Medical Sciences

${ }^{2}$ Punjab Institute of Medical Sciences

${ }^{3}$ Nilratan Sircar Medical College and Hospital

${ }^{4}$ NHS England

${ }^{5}$ Nepal Medical College Teaching Hospital

December 2, 2021

\begin{abstract}
Classic Congenital Adrenal Hyperplasia in males usually presents late until before puberty, but rarely at birth. In cases of undiagnosed $\mathrm{CAH}$, severe deficiency of cortisol may lead to life threatening situation in an infant with acute salt losing crisis. Screening for CAH at birth can lead to better outcomes.

Devastating Salt-wasting Crisis in a Four-month-old Male Child with Congenital Adrenal Hyperplasia, Highlighting the Essence of Neonatal Screening
\end{abstract}

Nagaspurthy Anugu Reddy ${ }^{1}$, Sucheta Sharma ${ }^{2}$, Mainak Das $^{3}$, Ashutosh Kapoor $^{4}$, Upasana Maskey ${ }^{*}$

1. Suraksha Women and Children Hospital, Hyderabad India (ORCID-0000-0002-2356-1623) Email- reddyspurthy@gmail.com

2. Punjab Institute of Medical Sciences, Jalandhar (ORCID- 0000-0001-9205-0889) Email- sharmasucheta1994@gmail.com

3. Nilratan Sircar Medical College, Kolkata India (ORCID- 0000-0001-6838-4678) Emailmainakusmle1999@gmail.com

4. NHS, UK (ORCID- 0000-0002-2898-2881) Email- ashu.kap89@gmail.com

5. Everest Hospital, Kathmandu, Nepal (ORCID- 0000-0002-3106-4037) Email- upasnamaskey@gmail.com

*Corresponding Author: Upasana Maskey

Key Message- Congenital Adrenal Hyperplasia although rare can present with a devastating salt-wasting crisis, virilization of females, and failure to thrive. Early diagnosis through screening at birth can yield better outcomes. This case highlights the need for mandatory screening in low and middle-income countries.

\section{ABSTRACT}

Background: Congenital Adrenal Hyperplasia $(\mathrm{CAH})$ is a rare condition usually referred to as a group of genetic disorders resulting due to a deficiency of steroid enzymes required by adrenal glands to produce 
cortisol and mineralocorticoid hormones. It has an autosomal recessive mode of inheritance and is further categorized into two types - Classic \& Non-Classic. Non-Classic CAH is a more common milder form that presents late after puberty. Classic $\mathrm{CAH}$, although more severe, is rare and detected at birth and is associated with the life-threatening adrenal crisis in both sexes and virilization of the external genitalia in females (46, $\mathrm{XX}$ ) patients whereas in males no overt abnormality of the external genitalia is present.

Case presentation: We present a case of a four-month-old male child with the classic form of CAH who was brought with complaints of loose stools, projectile non bilious vomiting, decreased urine output, and failure to feed for three days. The child had a clinical presentation of salt wasting with hypoglycemia and hyperpigmentation of his genitalia. The USG findings revealed increased Anteroposterior diameter of renal pelvis indicative of a growth in the suprarenal area. 17-hydroxyprogesterone (17-OHP) was found to be elevated confirming the diagnosis. He was treated with hydrocortisone with gradual improvement in his glucose and electrolytes. The patient was discharged home on replacement therapy consisting of oral prednisolone and fludrocortisone acetate and followed up as outpatient with significant improvement in the clinical findings.

Conclusion: The fact that the child was not screened for $\mathrm{CAH}$ at birth led to the critical consequences of the disease in this case. To prevent life-threatening adrenal crisis and help perform appropriate sex assignments for affected female patients, newborn screening (NBS) programs for the classical form of CAH should be made mandatory even in low and middle-income countries.

\section{KEYWORDS- CAH, Congenital Adrenal Hyperplasia, Neonatal Screening INTRODUCTION}

Congenital Adrenal Hyperplasia $(\mathrm{CAH})$ is a group of inherited disorders caused by genetic defects that hinder the production of adrenal hormones, cortisol, and aldosterone, either completely or at their normal rate. It may be caused by a deficiency of one of the five enzymes responsible for cortisol synthesis. These enzymes include $21 \alpha$-hydroxylase, 11 $\beta$-hydroxylase and 17 $\alpha$-hydroxylase/17,20-lyase, cholesterol 20,22 desmolase and $3 \beta$-hydroxysteroid dehydrogenase [1]. Adrenal steroidogenesis occurs by a series of steps facilitated by the zone-specific enzyme expression and different types of CAH interrupt this process at distinct branch points. Furthermore, there is a substitute pathway for the endogenous synthesis of androgens, which also plays a significant role in the causation of $\mathrm{CAH}[2,3]$. $\mathrm{CAH}$ is further categorized into two types - Classic \& Non-Classic. Classic CAH, although a more severe form, is rare and is usually detected at birth and is associated with the life-threatening adrenal crisis in both sexes and virilization of the external genitalia in 46, XX patients. Non-Classic CAH is a more common form that has a milder phenotype in which clinical problems are not obvious during the neonatal period or childhood and generally develops during adolescence or adulthood $[4,5]$. In Classic $\mathrm{CAH}$, the findings usually visible at birth include ambiguous genitalia in females due to excess male androgens, whereas in males no overt abnormality of the external genitalia is present. In $95 \%$ of the cases of CAH, the identified deficiency is of $21 \alpha$-hydroxylase enzyme [6]. This leads to impairment of cortisol and aldosterone production with excess production of androgens that leads to the aforementioned findings. We present a case of a four-month-old male child who presented with Classic CAH with signs of dehydration, malnutrition, and failure to thrive.

\section{CASE PRESENTATION}

A four-month-old male child was brought to the emergency department by the parents with complaints of loose stools, projectile non bilious vomiting, decreased urine output, and failure to feed for three days.

Birth History- The child was born at 37 weeks of gestation by assisted vaginal delivery (vacuum) with a birth weight of $2.5 \mathrm{~kg}$. A single dose of betamethasone was given nine hours before the birth of the child. He had cried immediately after birth and the APGAR score was five at one minute and eight at five minutes. Injection Vit $\mathrm{K}$ with Vit D3 oral drops was given after birth. Routine immunization as per the National Healthcare System (NHS) was done and breastfeeding was initiated in the hospital and he was exclusively breastfed to date. He was the first child of a non-consanguineous couple. There was no family history of any 
chronic or genetic diseases.

During the present visit at four months of age, the child looked dehydrated with sunken eyes and dry oral mucosa. On thorough clinical examination of the child, the weight was $3.5 \mathrm{~kg}$ and body length was $60 \mathrm{~cm}$. Mild to moderate fever (temp: $100^{\circ} \mathrm{F}$ ) and irritability were noted. The blood pressure was $70 / 36 \mathrm{mmHg}$, heart rate was $112 \mathrm{bpm}$, resp rate was $25 / \mathrm{min}, \mathrm{SpO} 2$ was $92 \%$ at room air, and $97 \%$ on two liters of oxygen. Examination of the respiratory system, cardiovascular and abdomen were within normal limits. The male genitalia were correctly identified, hyperpigmentation of the overlying skin was observed as shown in Fig 1. With the current body weight of $3.57 \mathrm{~kg}$ (i.e., below 10th percentile), the child was labeled under failure to thrive. The patient was admitted to the pediatric inpatient unit with a provisional diagnosis of acute gastroenteritis with dehydration and hypoglycemia for observation and further management.

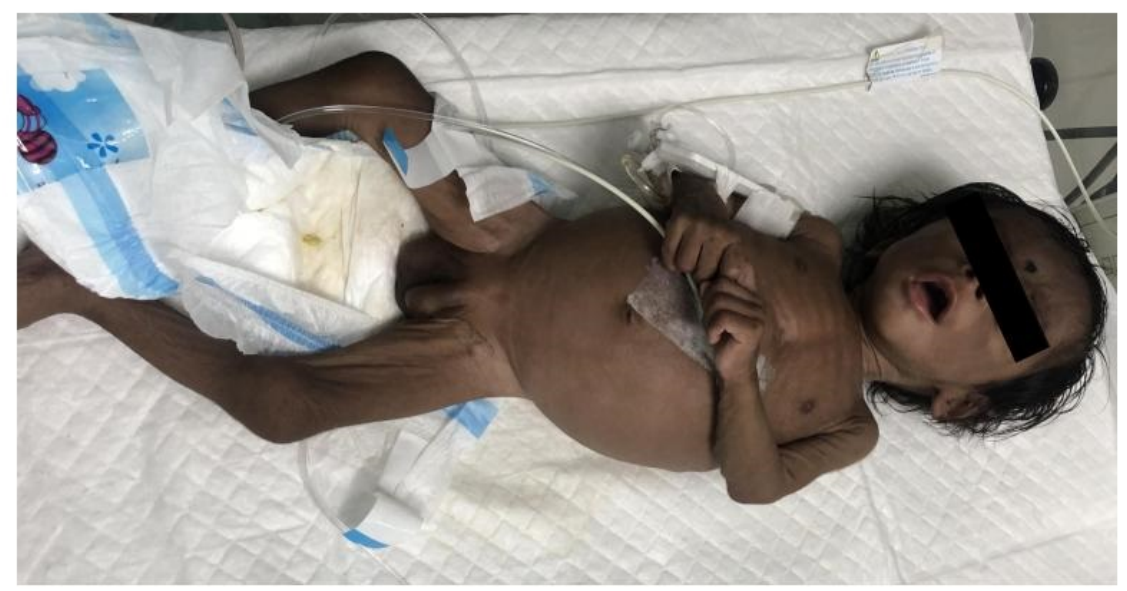

Figure 1 Male genitalia in a four-month-old infant showing hyperpigmentation.

Investigations showed deranged electrolytes with serum sodium: $119 \mathrm{mmol} / \mathrm{L}$, serum potassium: $1.7 \mathrm{mmol} / \mathrm{L}$, and serum creatinine: $61.89 \mu \mathrm{mol} / \mathrm{L}$. Random blood sugar was $0.89 \mathrm{mmol} / \mathrm{L}$. The hyponatremia and hypokalemia were assumed to be caused by dehydration owing to diarrhea and vomiting from the past three days. His initial blood gas analysis showed: pH- 7.54 (7.35-7.45), pCO2 $3.99 \mathrm{kPa}$ (4.27-6), HCO3 27.3 $\mathrm{mmol} / \mathrm{L}(22-28)$ suggestive of respiratory alkalosis. Normal levels of Magnesium $(0.74 \mathrm{mmol} / \mathrm{L})$ and ionized Calcium $(1.11 \mathrm{mmol} / \mathrm{L})$ successfully ruled out the possibility for Bartter and Gitelman syndrome. The lab results are outlined in Table 1. The stool for reducing substances was found to be negative. C-peptide was done to rule out insulinoma which was $0.15 \mathrm{ng} / \mathrm{ml}$. Liquid chromatography- tandem mass spectrometry (LC-MS/MS) was done to rule out any inborn metabolic errors, which reported to have no obvious organic aciduria.

Table 1: Laboratory findings

\begin{tabular}{|c|c|c|c|c|c|c|c|}
\hline Test & Value & & Normal range & Test & Value & & Normal range \\
\hline Hematology & & & & Arterial blood gas & & & \\
\hline WBC & 13.8 & 109cells/L & $(3.5-9.1)$ & $\mathrm{pH}$ & 7.54 & & $(7.35-7.45)$ \\
\hline $\mathrm{Hb}$ & 5.65 & $\mathrm{mmol} / \mathrm{L}$ & $(7.01-9.43)$ & $\mathrm{pCO} 2$ & 3.99 & $\mathrm{kPa}$ & $(4.27-6)$ \\
\hline Hct & 28 & $\%$ & $(33.4-44.9)$ & $\mathrm{HCO} 3$ & 27.3 & $\mathrm{mmol} / \mathrm{L}$ & $(22-28)$ \\
\hline Plt & 490 & 109cells/L & $(150-450)$ & & & & \\
\hline Biochemistry & & & & Endocrine & & & \\
\hline S.Cre & 61.89 & $\mu \mathrm{mol} / \mathrm{L}$ & $(35.37-123.79)$ & 17-OHP & 2.4 & $\mathrm{nmol} / \mathrm{L}$ & $(0.06-0.27)$ \\
\hline $\mathrm{Na}$ & 119 & $\mathrm{mmol} / \mathrm{L}$ & $(135-150)$ & Cortisol (8 am) & 60.7 & $\mathrm{nmol} / \mathrm{L}$ & $(165.5-634.5)$ \\
\hline
\end{tabular}




\begin{tabular}{|c|c|c|c|c|c|c|c|}
\hline K & 1.7 & $\mathrm{mmol} / \mathrm{L}$ & $(3.5-5.5)$ & Urine & & & \\
\hline $\mathrm{Cl}$ & 98 & $\mathrm{mmol} / \mathrm{L}$ & $(94-110)$ & $\mathrm{Na}$ & 36 & $\mathrm{mmol} / \mathrm{L}$ & $(40-220)$ \\
\hline Ca (Ionized) & 1.11 & $\mathrm{mmol} / \mathrm{L}$ & $(1.10-1.35)$ & K & 25 & $\mathrm{mmol} / \mathrm{L}$ & $(20-125)$ \\
\hline $\mathrm{Mg}$ & 0.74 & $\mathrm{mmol} / \mathrm{L}$ & $(0.62-0.82)$ & $\mathrm{Cl}$ & 72 & $\mathrm{mmol} / \mathrm{L}$ & $(100-250)$ \\
\hline T-bil & 7.01 & $\mu \mathrm{mol} / \mathrm{L}$ & $(3.42-18.81)$ & & & & \\
\hline $\mathrm{AST}$ & 0.93 & $\mu \mathrm{Kat} / \mathrm{L}$ & $(0.17-0.66)$ & & & & \\
\hline ALT & 0.43 & $\mu \mathrm{Kat} / \mathrm{L}$ & $(0.08-0.75)$ & & & & \\
\hline Glu (RBS) & 0.89 & $\mathrm{mmol} / \mathrm{L}$ & $(2.78-6.05)$ & & & & \\
\hline CRP & 101000 & $\mu \mathrm{g} / \mathrm{L}$ & $<6000$ & & & & \\
\hline
\end{tabular}

WBC: white blood cell; Hb: hemoglobin; Hct: hematocrit; Plt: platelet; S.Cre: serum creatinine; Na: sodium; K: potassium; Cl: chloride; Ca: calcium; Mg: magnesium; T-bil: total bilirubin; AST: aspartate aminotransferase; ALT: alanine aminotransferase; Glu (RBS): glucose (random blood sugar); CRP: c-reactive protein; 17-OHP: 17-hydroxyprogesterone.

X-ray erect abdomen was normal as shown in Fig 2. ECG was interpreted to be normal. Ultrasonography of the abdomen and pelvis (Fig $3.1 \& 3.2$ ) showed bilateral increased renal echotexture probably due to dehydration and prominent left renal pelvicalyceal system with an anteroposterior diameter of renal pelvis $11 \mathrm{~mm}$. The patient was given supportive measures and started on antibiotics to cover possible sepsis but the work-up for sepsis later came back negative. The electrolyte levels on day two showed Na-119 mmol/l, K-6.0 mmol/L. The morning sample for Cortisol drawn at 8 am on day two showed decreased levels of the hormone $(60.7 \mathrm{nmol} / \mathrm{L})$. Patient was started on Hydrocortisone $15 \mathrm{mg}$ IV eight hourly with hourly monitoring of vitals. The blood was drawn and sent for 17-hydroxyprogesterone (17-OHP) for suspicion of $\mathrm{CAH}$ which was found to be elevated $2.4 \mathrm{nmol} / \mathrm{L}$ (normal: 0.06-0.27) indicative of the diagnosis. His glucose and electrolytes were monitored daily while in the hospital, which gradually improved by the seventh day of hospitalization. Further clinical testing was refused by the parents of the child. After the improvement in clinical signs, the patient was discharged home on replacement therapy consisting of oral prednisolone and fludrocortisone acetate. He was followed up after seven days in the outpatient department. His weight had improved, electrolytes were normal and medications were adjusted according to his weight.

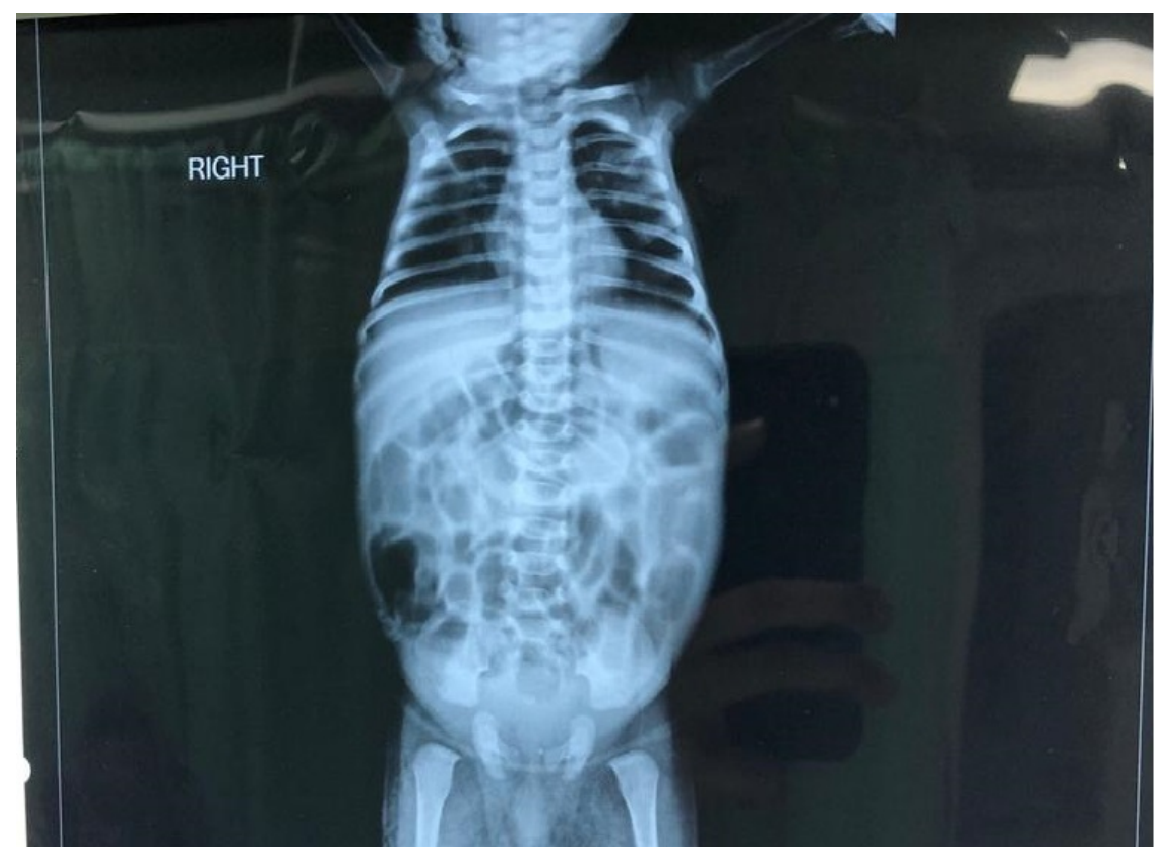


Figure 2 - X-ray erect abdomen showing normal findings

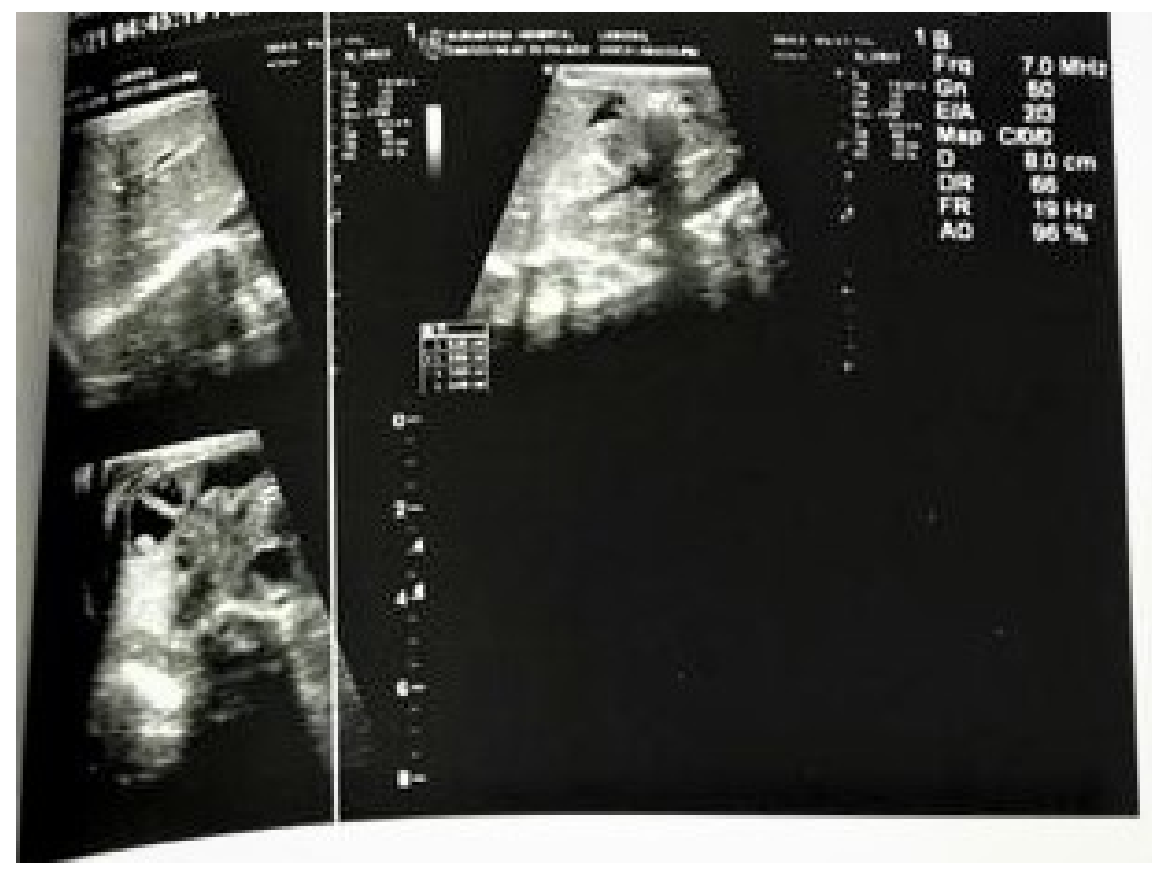

Figure 3.1 - USG showing bilateral increased renal echotexture

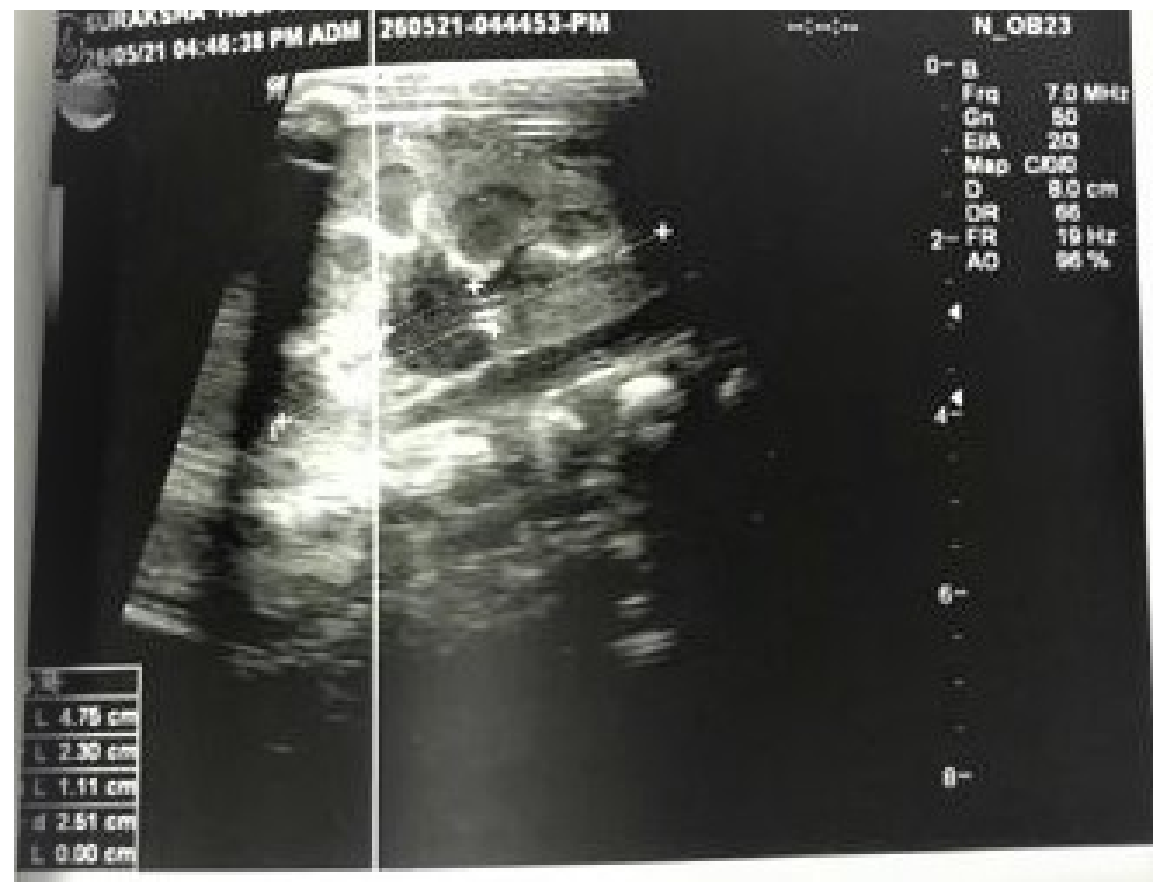

Figure 3.2 - Ultrasound showing a prominent left renal pelvicalyceal system with an $11 \mathrm{~mm}$ AP diameter of the renal pelvis

\section{DISCUSSION}


Classic congenital adrenal hyperplasia $(\mathrm{CAH})$ is generally caused by mutations in the CYP21A2 gene (resulting in 21 $\alpha$-hydroxylase deficiency) and has a prevalence of 1:15,000 [7]. The mode of inheritance is mainly autosomal recessive [8]. The patients deficient in 21?-hydroxylase present with signs of cortisol deficiency along with aldosterone deficiency in life-threatening situations. Insufficiency of adrenal hormones disturbs the balance of the hypothalamus-pituitary-adrenal (HPA) axis and impairs the negative feedback system leading to an increase in ACTH secretion from the pituitary gland. The stimulant effect of ACTH on the steroidogenesis pathway, in turn, leads to hyperplasia of both the adrenal glands with increased production of androgens. The intensity of clinical manifestations depends on the site and severity of the defect in the biosynthesis pathway [8]. In this variety, there is an accumulation of 17-OH-progesterone in the serum, which is further redirected to form adrenal androgens [8]. In about one-third of the cases, this defect is severe and presents at birth with features of glucocorticoid and mineralocorticoid deficiency and elevated androgens. The remaining two-third of the cases have adequate secretion of mineralocorticoids but there may be features of cortisol insufficiency and/or ACTH and androgen excess. Sometimes mild enzyme defects may be present that are not apparent until adult life, when females may present with amenorrhea and/or hirsutism, which is referred to as Late-onset CAH [8].

With the increasing concern on inherited defects, screening for CAH has been a part of the routine neonatal check-up in many parts of the world. In Sweden and Norway, the test is performed by measuring 17 hydroxyprogesterone (17-OHP) in blood using a filter paper no earlier than 48 hours after the birth. The other screening methods are radioimmunoassay employed in the US, enzyme-linked immunosorbent assay in Japan, and time-resolved fluoroimmunoassay in Europe. Preterm newborns have a higher 17-OHP concentration in serum than babies born at term. Thus, cut-off levels are based on gestational age in Japan and Europe, and birth weight in the US [9]. Screening aims to determine the sex, improve the outcomes and prevent neonatal deaths [10]. During the prenatal period methods like amniocentesis and chorionic villous sampling (CVS) can be utilized to screen the fetus for genetic defects. The lack of adequate screening in this part of the world led to the severe clinical presentation in this child with typical signs of salt loss and hypoglycemia. It has been shown that these findings can have a negative influence on the child's cognitive performance. The long-term consequences of the salt-wasting crisis at birth are hard to predict, but early diagnosis and avoiding hypo-cortisolism in the neonatal period has proved to improve prognosis and lead to a favorable cognitive development as well [7].

The management of this disorder is a constant balancing act. Although the prenatal administration of synthetic glucocorticoid (dexamethasone) to pregnant females with a previous history of a child with CAH has been shown to ameliorate virilization of external genitalia in the affected female fetus, uncertainties exist in terms of long-term efficacy and safety profile of this measure or its use in male fetuses. Adding to the concern is the fact that the dose of dexamethasone that the fetus is exposed to is estimated to be 60 times the normal fetal cortisol level [12]. The management involves the lifelong substitution of cortisol but due to the inability to match the exact circadian rhythm of the hormones, it carries a risk of both under and over-treatment. Our patient improved significantly with the appropriate dose and treatment with normalization of the electrolytes and adequate weight gain. But a careful approach was warranted as the receptors for both glucocorticoids and mineralocorticoids are overly expressed in parts of the brain like the hippocampus, amygdala, and prefrontal cortex and excess replacement may disturb the cognitive and affective functions in patients receiving overtreatment [7]. Ideally, there should be a balance between the substitution and suppression of glucocorticoids and mineralocorticoids. Needless to say, patients with Classic CAH on replacement therapy require added care and monitoring especially during the critical stages of any illness. Administration of stress doses of hydrocortisone acutely during sickness undoubtedly plays a very crucial role but may still be unable to control hypoglycemia. Although our patient showed significant improvement in the glucose levels with the treatment, as per the guidelines, additional glucose supplementation is essential in preventing a hypoglycemic crisis, especially in the pediatric population [11].

The potentially fatal presentation of the patient at the time of arrival to the hospital could have been prevented, had the child been screened for the disorder at birth. This case, therefore, highlights the significance of neonatal screening for $\mathrm{CAH}$ at birth for better outcomes in patients. The newborn screening for CAH has 
already been a routine part of the neonatal screening protocol for most countries [12] and calls for an urgent need in low and middle-income countries as well.

\section{CONCLUSION}

Classic congenital adrenal hyperplasia, although rare, can present with a devastating picture of hypoglycemia and salt-wasting crisis as seen in this case. Although female patients present with signs of virilization, this male child presented with hyperpigmentation of his genitalia with additional concerns of failure to thrive. There is an increased risk for cognitive impairment as a consequence of the presentation seen with this disorder and thus timely diagnosis and management are crucial. This case highlights the need for neonatal screening for the $\mathrm{CAH}$ which is currently not a mandatory practice in some countries. Early diagnosis through screening at birth can contribute to better outcomes and prognosis.

\section{DATA AVAILABILITY}

The literature review data used to support the findings of this study are included within the article.

\section{DISCLOSURE}

The content of this publication is solely the responsibility of the authors and does not necessarily represent official views of the institutions the author belongs to.

\section{CONFLICT OF INTEREST}

The authors declare that they have no conflicts of interest.

\section{FUNDING INFORMATION}

There was no funding issued by any organization in the support of submitted work.

\section{AUTHOR CONTRIBUTION STATEMENT}

All authors have contributed equally in the submitted article. The final draft was reviewed and approved for submission by each contributing author.

References 1.Honour, J. W. (2009). Diagnosis of Diseases of Steroid Hormone Production, Metabolism and Action. Jor 